\title{
Relationship Between Steroid Hormones and Metabolic Profile in Women With Polycystic Ovary Syndrome
}

\section{LAZÚROVÁ ${ }^{1}$, Z. LAZÚROVÁ ${ }^{2}$, J. FIGUROVÁ ${ }^{1}$, S. UJHÁZI ${ }^{1}$, I. DRAVECKÁ ${ }^{1}$, J. MAŠLANKOVÁ ${ }^{3}$, M. MAREKOVÁ}

${ }^{1}$ First Department of Internal medicine, Medical Faculty of P. J. Šafarik University, Košice, Slovakia, ${ }^{2}$ Fourth Department of Internal medicine, L. Pasteur University Hospital, Košice, Slovakia, ${ }^{3}$ Department of Biochemistry, Medical Faculty of P. J. Šafarik University, Košice, Slovakia

Received October 21, 2018

Accepted January 25, 2019

Epub Ahead of Print March 22, 2019

\section{Summary}

Polycystic ovary syndrome (PCOS) is commonly associated with a higher cardiometabolic risk. The relationship between steroid hormones and cardiometabolic profile in PCOS has been evaluated, but no single hormonal predictor of this association has been identified to determine. To determine the relationship between steroid hormones and cardiometabolic risk factors in PCOS women. Study included 64 women diagnosed with PCOS. Fasting blood samples were analyzed for biochemical, metabolic parameters and sex steroid hormones. PCOS women with $B M I \geq 27$ had significantly higher serum free testosterone (FT), free androgen index (FAI), estrone (E1) $(p=0.014, p=0.02$, $p=0.01$ ) than those with normal weight. In all subjects E1 positively correlated with BMI $(p=0.0067)$, serum insulin $(p=0.0046)$, HOMA-IR $(p=0.0125)$ and negatively with HDL-cholesterol $(p=0.009)$. FAI positively correlated with serum cholesterol $(p=0.0457)$, triacylglycerols (TAG) $(p=0.0001)$, HOMA-IR $(p=0.037)$, and glycemia $(p=0.0001)$, negatively with HDL-cholesterol $(p=0.029)$. In multiple linear regression model E1 most significantly predicted HOMA-IR, whereas FT/FAI predicted HDL-cholesterol and BMI. We conclude that PCOS women with marked overweight or obesity have higher FT, FAI and E1 as compared with nonobese PCOS subjects. E1 and FT may predict worse cardiometabolic profile in PCOS.

\section{Key words}

Polycystic ovary syndrome • Sex steroids • Cardiometabolic risk • Free testosterone • Estrone

\section{Corresponding author}

I. Lazúrová, First Department of Internal Medicine, Medical Faculty of P.J. Šafarik University Košice, Trieda SNP 1, 04011 Košice, Slovakia. Fax: +421 44234 3411. E-mail: ivica.lazurova@upjs.sk

\section{Introduction}

Polycystic ovary syndrome (PCOS) is a common endocrine disease with a wide phenotype variability affecting approximately 5-15\% of reproductive-aged women (March et al. 2010). Based on Rotterdam criteria, it is defined by two of the following three features: chronic oligo/anovulation, hyperandrogenic state (clinical and/or biochemical) and polycystic ovarian morphology (PCOM) detected by ultrasound (Rotterdam ESHRE/ASRM 2004). There is evidence that PCOS is commonly associated with metabolic syndrome (MS) or its components and increased prevalence of cardiometabolic risk factors has been consistently reported. Numerous studies demonstrated that PCOS women have higher prevalence of obesity, arterial hypertension, impaired glucose metabolism and dyslipidemia in comparison to controls (Alexander et al. 2009, Azziz et al. 2009). Data suggest 80-100\% increased risk of MS in PCOS women according to diagnostic criteria (Yildiz et al. 2012). However, the relationship between metabolic parameters and sex steroid hormone production is still not fully understood. 
It is well known, that dyslipidemia, insulin resistance or type 2 diabetes mellitus (T2DM) and obesity are more prevalent in PCOS women with clinical or biochemical hyperandrogenism (The Amsterdam ESHRE/ASRM 2012, Sirman et al. 2014). Changes in insulin pulsatility and insulin clearance, as well as beta cell dysfunction in PCOS were demonstrated not only in obese but also in lean patients. (Grimmichova et al. 2008, Torchen 2017). In previous studies free testosterone (FT), free androgen index (FAI) and estrone (E1) significantly predicted PCOS in women of all ages, whereas androstenedione (A4) was associated with more severe phenotype in PCOS women (Stener-Victorin et al. 2010, Conway et al. 2014).

Sex steroid hormones are recognized to play a crucial role in the development of cardiovascular diseases. The relationship between sex steroid hormones and cardiometabolic profile in the general population and PCOS women has also been evaluated, but no single and definitive humoral or endocrine marker either for PCOS or for its association with MS has been clearly identified until this time. Studies in both sexes have proposed that extremely low and high androgen levels are associated with an increased cardiovascular disease (CVD) risk (Laughlin et al. 2010, Soisson et al. 2013). PCOS is commonly presented with changes of several sex steroids and there is a raising question, which of these hormones are associated with CVD risk factors. Current data on association of various sexual steroids with body weight, glucose tolerance and lipid profile or prediction of cardiovascular risk by sexual steroid hormones are limited and controversial. We hypothesized that sex steroids with marked relationship to adipose tissue which are increased in PCOS women could predict worse cardiometabolic profile in these patients.

The aim of the study was primarily to compare metabolic parameters and the levels of sex steroids between the group of non obese PCOS women and women with PCOS and marked overweight or obesity. The second aim of the study was to determine the relationship of wide spectrum of sex steroids to cardiometabolic risk in patients with PCOS.

\section{Subjects and Methods}

\section{Subjects}

The study included 64 Caucasian women diagnosed with PCOS. Mean age of the patients was $28.9 \pm 5$ years (range 22-43, median 28 years). The diagnosis of PCOS was based on the Rotterdam criteria. Clinical hyperandrogenism was defined as the presence of hirsutism (modified Ferriman-Galwey score $\geq 6$ ), acne or androgenic alopecia, biochemical hyperandrogenemia was postulated if serum FT levels reached $1.1 \mathrm{ng} / \mathrm{ml}$ or FAI higher than $8 \%$. Chronic anovulation was defined by menstrual cycle of less than 21 or more than 35 days, simultaneously with progesterone levels $6 \mathrm{ng} / \mathrm{ml}$ and less on days 20.-23. of the menstrual cycle in two consecutive cycles. PCOM was defined as the presence of 12 or more ovarian follicles on ultrasound or ovarian volume larger than $10 \mathrm{ml}$.

Exclusion criteria were congenital adrenal hyperplasia, Cushing's syndrome, prolactinomas and androgen secreting tumors. All patients had normal thyroid, liver and kidney function and they did not take any medication that could influence the hypothalamicpituitary-ovarian axis during the last six months.

All subjects signed a written informed consent and the study was approved by the Ethical committee of the L. Pasteur University Hospital in Košice, Slovakia and fulfilled the ethical guidelines of the most recent declaration of Helsinki.

All patients were divided according to body mass index (BMI) into two groups. The first group consisted of 34 patients with $\mathrm{BMI}<27 \mathrm{~kg} / \mathrm{m}^{2}$, the second of 30 patients with $\mathrm{BMI} \geq 27 \mathrm{~kg} / \mathrm{m}^{2}$.

\section{Methods}

Weight, height and waist circumference (WC) were measured in all subjects. Weight and height were used to calculate BMI, which was assessed by dividing weight by height squared $\left(\mathrm{kg} / \mathrm{m}^{2}\right)$. Fasting blood samples were collected at approximately 7.00 a.m. in an early follicular phase, i.e. between days 3-7 of the menstrual cycle, or whenever in those with amenorrhea. Anthropometric data were obtained in the same day. Blood samples were used to analyze serum glucose, insulin and lipid profile - total cholesterol (T-C), LDL-cholesterol (LDL-C), HDL-cholesterol (HDL-C), triglycerides (TG).

Circulating levels of follicle-stimulating hormone (FSH), luteinizing hormone (LH), sexual hormone binding protein (SHBG), total testosterone (TT), FT, dihydrotestosterone (DHT), dehydroepiandrosterone sulfate (DHEAS), androstenedione (A4), E1 and estradiol (E2) were evaluated from the same fasting blood sample. FAI was calculated as TT x 100 / SHBG. The HOMA-IR (homeostasis model assessment insulin 
resistance) was assessed by equation: HOMA-IR = (glucose $\mathrm{x}$ insulin) / 22.5.

Serum glucose and lipids were analyzed routineously using autoanalyzer (Roche Diagnostics $\mathrm{GmbH}$ ). Hormonal levels of SHBG, LH, FSH, DHEAS and E2 were determined using chemiluminescent immunoassay - CLIA (analyzer ARCHITECT, module C, Abbott, USA). The analytical sensitivity of the ARCHITECT E2 assay is $\leq 10 \mathrm{pg} / \mathrm{ml}$, the functional sensitivity is $\leq 25 \mathrm{pg} / \mathrm{ml}$. The analytical sensitivity for DHEAS was calculated to be $0.18 \mu \mathrm{g} / \mathrm{ml}$ at a $95 \%$ level of confidence. The specificity for DHEAS assay is designed to have $\leq 10 \%$ cross-reactivity when tested with structurally similar compounds. TT, FT and A4 were detected by radio-immunoassay using commercially available kits of DIA Source (Belgium).

Analytes measured by ELISAs with catalog numbers were E1 (DE4174) and DHT (DE2330) from Demeditec diagnostics GmbH. The intra-assay and interassay precision $\mathrm{CV} \%$ for human serum immunoassay were: for DHT (3.9-11.4) and (5.9-12.1), for E1 (6.5-9.5) and (12.8-14.8). The sensitivity was: for DHT $6 \mathrm{pg} / \mathrm{ml}$ and for E1 $2.21 \mathrm{pg} / \mathrm{ml}$. The cross-reactivity between all tested analytes was less than $0.01 \%$.

\section{Statistical analysis}

SAS JMP version 13.0.0 (USA) software was used for statistical analysis. Data are presented as mean \pm SD. For normally distributed variables Student's $t$-test was used to compare means between groups, whereas for non-normally distributed data the non-parametric Mann Whitney test was used. Linear regression analysis was used to assess correlations between variables. Values were considered statistically significant at $\mathrm{p} \leq 0.05$. Multiple linear regression analysis was used with various metabolic parameters as dependent variables and age, BMI, steroid hormones as independent variables. The power of significance is expressed as Log Worth, which was calculated as follows: $-\log 10$ (p-value), where the adjusted $p$-value is calculated in a complex manner that takes into account the number of different ways splits can occur. A value that exceeds 2 was considered statistically significant.

\section{Results}

Measured anthropometric data, biochemical and hormonal variables in the group of PCOS women and subgroups according to BMI are presented in the Table 1.
As expected, PCOS women with $\mathrm{BMI} \geq 27 \mathrm{~kg} / \mathrm{m}^{2}$ had significantly higher insulin levels and HOMA-IR, they also had tendency to a higher fasting glycemia level. This group of patients had higher serum T-C and LDL-C levels, slightly higher TG (borderline significance). PCOS women with $\mathrm{BMI} \geq 27 \mathrm{~kg} / \mathrm{m}^{2}$ demonstrated significantly higher FT, FAI, E1 and lower SHBG levels. There was found a moderately higher serum DHT level with borderline significance $(p=0.06)$ in the group of PCOS women with higher BMI. There were no significant differences in ASD and DHEAS levels as well as TT levels between both groups (Table 1).

There was positive correlation between BMI and E1 ( $p=0.0067, r=0.34)$ (Fig. 1), positive correlation between BMI and FT with borderline significance $(\mathrm{p}=0.09, \mathrm{r}=0.22), \mathrm{BMI}$ and FAI $(\mathrm{p}=0.07, \mathrm{r}=0.26$, $)$, respectively (Fig. 2). FAI positively correlated with total cholesterol $(\mathrm{p}=0.0457, \mathrm{r}=0.3), \mathrm{TG}(\mathrm{p}=0.0001, \mathrm{r}=0.53)$, HOMA-IR ( $p=0.037, r=0.36)$, serum insulin $(p=0.0428$, $\mathrm{r}=0.35$, $)$ and glycemia $(\mathrm{p}=0.0001, \mathrm{r}=0.6)$ and negatively correlated with HDL-C ( $\mathrm{p}=0.029, \mathrm{r}=-0.33)$, respectively (Fig. 2). In addition, we observed that serum E1 levels were in significant negative correlation with HDL-C $(\mathrm{p}=0.0099, \mathrm{r}=-0.36)$ and in positive correlation with serum insulin level $(\mathrm{p}=0.0046, \mathrm{r}=0.4)$ as well as HOMA-IR $(\mathrm{p}=0.0125, \mathrm{r}=0.39) \quad$ (Fig. 1). Other sex steroids, such as E2, TT, A4, DHEAS and DHT were not in significant relationship with metabolic parameters (BMI, waist circumference, glycemia, insulin, HOMA-IR, T-C, TG, LDL-C, HDL-C).

In multiple linear regression analysis model estrone was the most significantly related to insulin resistance expressed as HOMA-IR (LW 2.913, $\mathrm{p}=0.0012$ ) even after adjustment for BMI (LW 2.422, $\mathrm{p}=0.00396$ ). After adjustment for BMI, HDL-C was most significantly predicted by FT (LW 2.25, p=0.05). Moreover, only FT significantly predicted BMI (LW 2.196, $\mathrm{p}=0.006$ ) among all steroid hormones.

\section{Discussion}

The primary aim of the study was to compare metabolic parameters and the levels of sex steroids between non obese PCOS women and those with marked overweight or obesity (BMI $\left.\geq 27 \mathrm{~kg} / \mathrm{m}^{2}\right)$. The second aim was to determine relationship of steroid hormones to selected components of the MS using a multiple linear regression model. Decrease in HDL-C and increase in TG levels are well known lipid profile characteristics in 
Table 1. Anthropometric, biochemical and hormonal variables in PCOS women divided according to BMI.

\begin{tabular}{|c|c|c|c|c|}
\hline Parameter & $\begin{array}{l}\text { PCOS } \\
\text { all group } \\
(n=64)\end{array}$ & $\begin{array}{c}\text { PCOS } \\
\text { BMI }<27 \mathrm{~kg} / \mathrm{m}^{2} \\
(\mathbf{n}=\mathbf{3 4})\end{array}$ & $\begin{array}{c}\text { PCOS } \\
\text { BMI } \geq 27 \mathrm{~kg} / \mathrm{m}^{2} \\
(\mathrm{n}=\mathbf{3 0})\end{array}$ & p value \\
\hline Age (years) & $28.9 \pm 5$ & $27 \pm 5$ & $30.7 \pm 5.2$ & 0.011 \\
\hline$B M I\left(\mathrm{~kg} / \mathrm{m}^{2}\right)$ & $27.7 \pm 7$ & $22.3 \pm 2.3$ & $34.2 \pm 5.4$ & 0.0000 \\
\hline Waist (cm) & $84 \pm 18$ & $72 \pm 7$ & $100 \pm 15$ & 0.0000 \\
\hline Glycemia (mmol/l) & $5.1 \pm 1.5$ & $4.7 \pm 0.4$ & $5.5 \pm 2.2$ & 0.0731 \\
\hline Insulin (mIU/l) & $14.4 \pm 9.7$ & $9.1 \pm 5.1$ & $21.5 \pm 9$ & 0.0000 \\
\hline$H O M A-I R$ & $3.2 \pm 2.5$ & $1.9 \pm 1.1$ & $4.9 \pm 2.7$ & 0.00026 \\
\hline Cholesterol (mmol/l) & $4.9 \pm 1.1$ & $4.6 \pm 1$ & $5.3 \pm 1.26$ & 0.03 \\
\hline$T G(\mathrm{mmol} / \mathrm{l})$ & $2.1 \pm 4.8$ & $1.02 \pm 0.5$ & $3.3 \pm 6.9$ & 0.09 \\
\hline$H D L-C(\mathrm{mmol} / \mathrm{l})$ & $1.5 \pm 0.4$ & $1.7 \pm 0.3$ & $1.23 \pm 0.3$ & 0.0000 \\
\hline$L D L-C(\mathrm{mmol} / \mathrm{l})$ & $2.7 \pm 0.8$ & $2.5 \pm 0.75$ & $2.9 \pm 0.77$ & 0.03 \\
\hline FSH (IU/l) & $4.2 \pm 1.6$ & $4.5 \pm 1.6$ & $3.8 \pm 1.4$ & 0.11 \\
\hline LH (IU/l) & $8.6 \pm 7.1$ & $10.0 \pm 8$ & $7.0 \pm 4.7$ & 0.121 \\
\hline$T T(n g / m l)$ & $1.1 \pm 0.38$ & $1.03 \pm 0.35$ & $1.1 \pm 0.3$ & 0.321 \\
\hline$F T(p g / m l)$ & $4.3 \pm 1.2$ & $3.9 \pm 1.1$ & $4.7 \pm 1.2$ & 0.014 \\
\hline SHBG (nmol/l) & $58.7 \pm 65$ & $79.7 \pm 80$ & $36.7 \pm 36.5$ & 0.016 \\
\hline$F A I$ & $11.3 \pm 11.1$ & $7.6 \pm 7.8$ & $14.8 \pm 12.9$ & 0.02 \\
\hline$A 4(n g / m l)$ & $5.0 \pm 1.4$ & $4.8 \pm 1.4$ & $5.2 \pm 1.5$ & 0.42 \\
\hline DHEAS $(\mu \mathrm{g} / 100 \mathrm{ml})$ & $322 \pm 164$ & $306 \pm 137$ & $332 \pm 194$ & 0.619 \\
\hline$E 2(\mathrm{pg} / \mathrm{ml})$ & $64.5 \pm 42.1$ & $75.12 \pm 43.7$ & $58.5 \pm 39.7$ & 0.09 \\
\hline$E 1(\mathrm{pg} / \mathrm{ml})$ & $114.2 \pm 129$ & $78.3 \pm 75$ & $161 \pm 165$ & 0.01 \\
\hline$D H T(p g / m l)$ & $683 \pm 449$ & $587 \pm 267$ & $808 \pm 596$ & 0.06 \\
\hline
\end{tabular}

BMI - body mass index, HOMA-IR - homeostatic model assessment insulin resistance, TG - triglycerides, HDL-C - high density lipoprotein cholesterol, LDL-C - low density lipoprotein cholesterol, FSH - follicle stimulating hormone, LH - luteinizing hormone, TT - total testosterone, FT - free testosterone, SHBG - sex hormone binding globulin, FAI - free androgen index, A4 - androstenedione, DHEAS - dehydroepiandrosterone sulphate, E2 - estradiol, E1 - estrone, DHT - dihydrotestosterone.

PCOS women (Legro et al. 2011, Scicchitano et al. 2012, Kim and Choi 2013). For this study HDL-C has been used as a predictor of CVD risk.

As expected, $\mathrm{PCOS}$ women with $\mathrm{BMI} \geq 27 \mathrm{~kg} / \mathrm{m}^{2}$ had significantly worse metabolic profile and higher levels of FT, FAI and E1 in comparison to nonobese group. We also detected slightly higher DHT in obese group with borderline significance. There were no differences in the levels of TT, A4 and DHEAS between both groups. Numerous studies have demonstrated that PCOS women especially those with higher BMI are at increased cardiovascular risk and that metabolic abnormalities are more prevalent in obese PCOS women (Legro 2012, Daan et al. 2015, Layegh et al. 2016). Results of the current study are in agreement with published data, showing higher CVD risk in obese PCOS patients and more severe hyperandrogenic state in
PCOS women with higher BMI (Azziz et al. 2009).

In general, many studies demonstrated higher serum levels of ovarian and adrenal androgens, estrogens, sex steroid precursors and glucuronidated androgen metabolites in PCOS women in comparison with non PCOS controls (Stener-Victorin et al. 2010, Nehir et al. 2016). In the Japanese study, abnormal values of FT, FAI and E1 were the most common laboratory findings among all sexual steroids (The Japanese Society 2007, Kubota 2013). In another study, serum E1 and FT were independently associated with PCOS. Combination of elevated E1 and FT was able to discriminate PCOS and non PCOS women with high sensitivity and specificity (Stener-Victorin et al. 2010). In the study of Pinola et al. (2015), FT, FAI and ASD were the best predictive factors for having PCOS. 

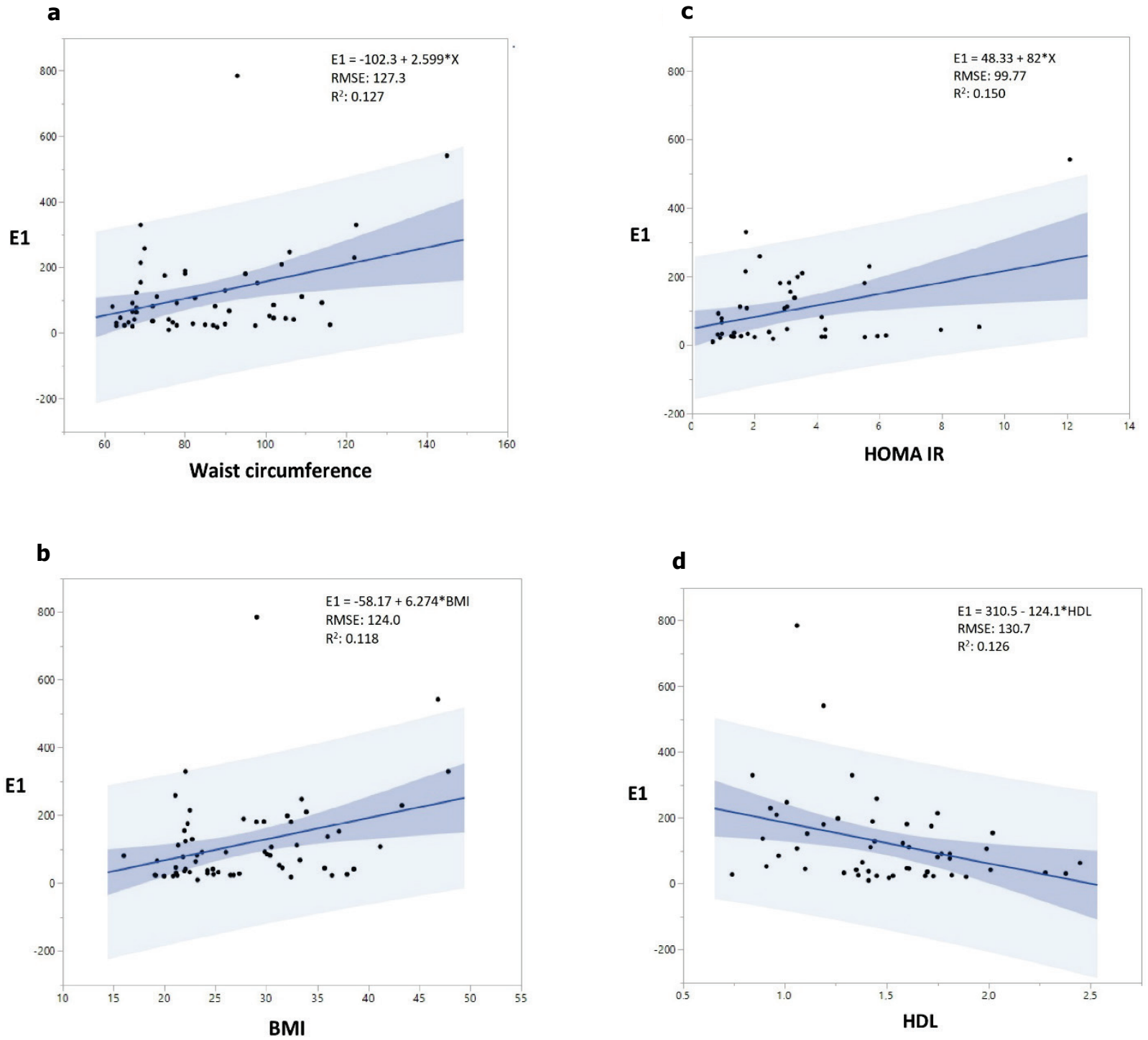

Fig. 1. Linear regression analysis models show significant positive correlations between E1 and waist circumference (a), BMI (b), HOMA-IR (c) and negative correlation between E1 and HDL-C (d).

Unfortunately, there is not known, which steroid hormone is the main predictor of the worse metabolic profile in PCOS women. Although, some studies demonstrated more hyperandrogenic profile in obese PCOS women, we found the higher serum levels of FT, FAI and slightly higher DHT in obese PCOS women. Similarly to Layegh et al. (2016) we did not detect significant differences in TT and A4 levels between obese and non obese subjects. In contrast with this study we did not find a difference in DHEAS levels between both groups according to BMI (Layegh et al. 2016). There was significant positive correlation between BMI and E1, borderline positive correlation between BMI and FT/FAI. FAI positively correlated with T-C, TG, HOMA-IR and negatively with HDL-C. Similar findings were observed in some other studies (Ebrahimi-
Mamaghani et al. 2015, Daan et al. 2015, Kische et al. 2016) showing that PCOS women with elevated FT levels have an adverse metabolic phenotype (Lerchbaum et al. 2014). The same was demonstrated in FAI which correlated more tightly with degree of obesity than with PCOS itself (Torchen 2017).

This study did not demonstrate a difference in serum DHEAS between obese and non obese PCOS women. We also did not find any relationship between DHEAS and metabolic parameters contrary to Moran et al. (2015) and Layegh et al. (2016). Thus we did not confirm some previous data that DHEAS levels are related to a better metabolic profile in PCOS women (Dong et al. 2015, Guducu et al. 2015, Amato et al. 2015). 

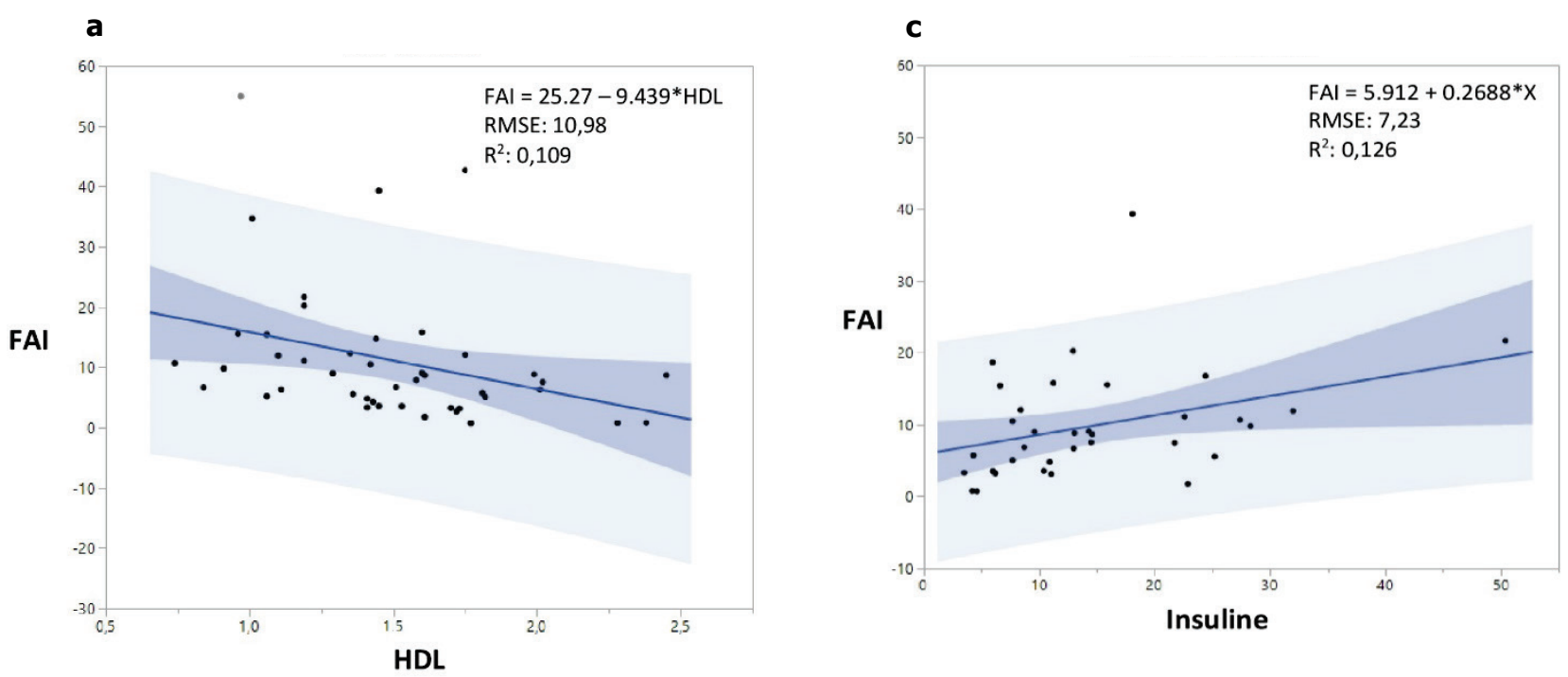

\section{b}
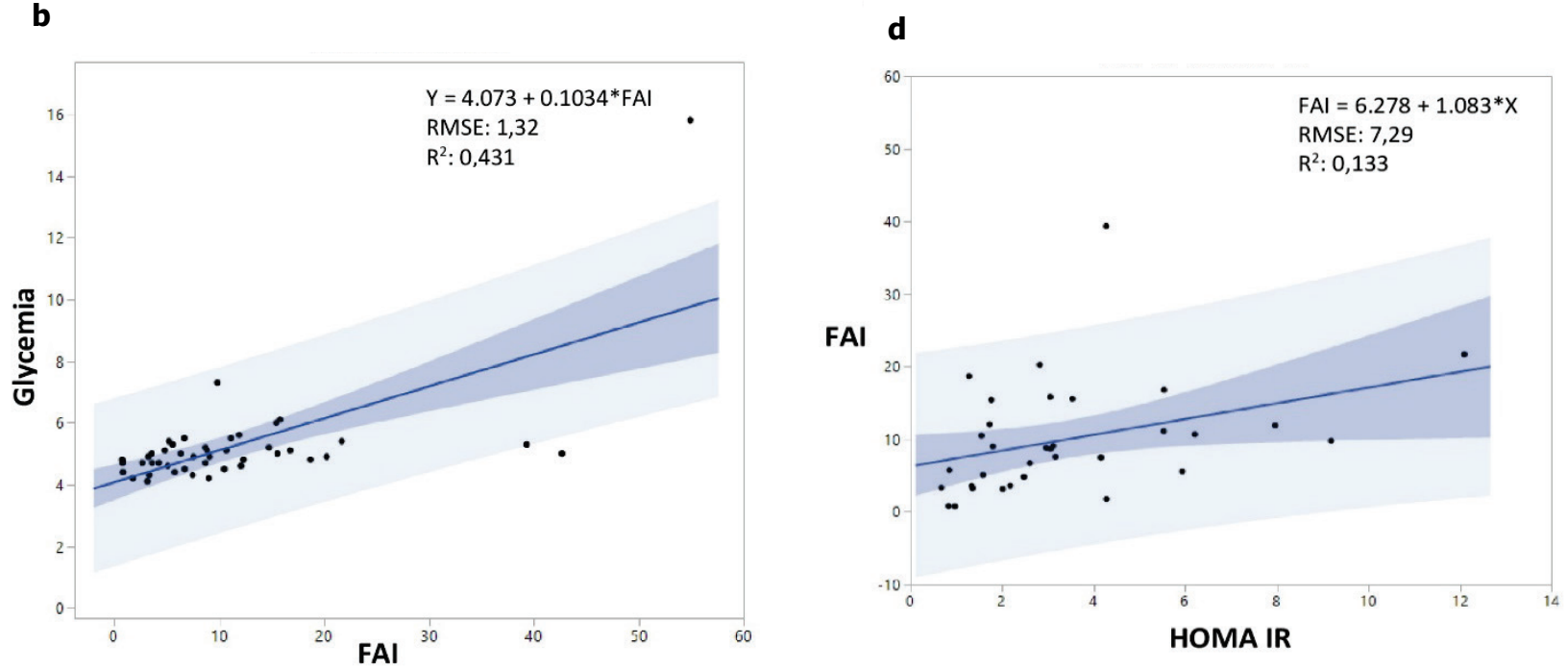

Fig. 2. Linear regression analysis models show negative correlation between FAI and HDL-C (a) and positive correlations between FAI and glycemia (b), insulin (c) and HOMA-IR (d).

Although few studies demonstrated enlargement of the adrenal gland and adrenal androgen excess in PCOS women (Azziz et al. 2013, Unlu et al. 2016), not all of them have found association of DHEAS with better metabolic profile. In particular, it can be explained by various cut off values for BMI in various studies and by heterogeneity of patients in examined groups. Based on these data, DHEAS appears to be a protective against CVD risk in PCOS women, but it's role in modulating CVD risk in PCOS women is still unknown (Godarzi et al. 2015, Alpanez et al. 2015).

In this study we did not demonstrate any relationship between A4 and metabolic profile in PCOS. In one study, A4 was related to more severe phenotype of the PCOS, others confirmed its association with better cardiometabolic profile (Moran et al. 2015,
Georgopoulos et al. 2014). Data from the literature are still poor and further studies are needed.

There are only few studies assessing the role of DHT in cardiometabolic risk in PCOS women. In the previous study, DHT was significantly higher in PCOS group (Stener-Victorin et al. 2010). Unfortunately, this study did not determine its relationship to BMI and CVD risks. In our study, DHT was slightly increased in PCOS patients with higher BMI with the borderline significance. This finding does not allow to postulate reliable conclusion and requires further observations.

Aside from androgens, endogenous estrogens have also been extensively studied as a potential predictor of CVD risk. In the study of Daan et al. (2015) adjustment of E2 attenuated association between FAI and CVD risk factors, however in another study E2/T ratio 
was significantly lower in PCOS subjects and negatively correlated with BMI (Chen et al. 2015). This study did not find any differences in E2 levels according to BMI. Simultaneously, we did not find any correlation of E2 with metabolic characteristics in PCOS women. Whereas, Kische et al. (2016) found a positive correlation of E2 with BMI, others did not confirm this association.

On the other hand, the levels of E1 are reported to be elevated in PCOS women and also in those with higher BMI. Many similar studies did not determine serum estrone levels, which is the main advantage of this study. There is an evidence that E1 is also increased in obese women regardless on the presence of PCOS (Nehir-Aytan et al. 2015, Mather et al. 2015). Previous study showed that incidence of T2D was directly associated with serum estrogens, especially E1 (Mather et al. 2015). In our cohort, E1 together with FT and FAI significantly correlated with majority of metabolic parameters. No other steroids were found to be in relationship with cardiometabolic risk. In multiple regression analysis E1 predicted insulin resistance even after age and BMI adjustment, that is the main priority of the study. Some studies have found that women with PCOS have increased total and also abdominal fat (Torchen 2017). Because E1 mostly reflects fat amount, we suggest that its higher levels and association with cardiometabolic risk are done via fat mass, which is the most important source of E1 production.

One of the strengths of this study is that we measured a wide spectrum of sex steroids, more than were measured in previous studies. This study has also several limitations. Main limitation is the fact, that we did not measure sex steroids levels using mass spectrometry. However, the high sensitivity and specificity of laboratory assays allows to postulate serious results. The second limitation is a relatively small group of patients enrolled in the study.

Despite limitations we can conclude that PCOS women with marked overweight and obesity have significantly worse cardiometabolic profile than those with BMI $<27 \mathrm{~kg} / \mathrm{m}^{2}$. PCOS group with higher BMI had significantly higher values of FT, FAI and E1, as well as slightly higher DHT. Among all sex steroid hormones, FT and E1 positively correlated with cardiometabolic risk characteristics, whereas other steroids were not in significant relationship to various components of metabolic syndrome. Because majority of PCOS women especially those with overweight or obesity have increased cardiometabolic risk and approximately $50 \%$ increase in cardiovascular disease deaths, all these women should be stratified and followed up for risk factors. The values of FT, FAI and E1 seem to be a suitable markers for follow-up strategy in these patients.

\section{Conflict of Interest}

There is no conflict of interest.

\section{Acknowledgements}

We thank the study participants whose continued dedication and commitment make this work possible. This research was supported by the Slovak Scientific Agency VEGA 1/0364/17.

\section{References}

ALEXANDER CJ, TANGCHITNOB EP, LEPOR NE: Polycystic ovary syndrome: a major unrecognized cardiovascular risk factor in women. Rev Obstet Gynecol 2: 232-239, 2009.

ALPANEZ M, LUQUE-RAMIREZ M, MATINEZ-GARCIA MA, FERNANDEZ-DURAN E, ALVAREZ-BIASCO F, ESCOBAR-MOREALE HF: Influence of adrenal hyperandrogenism on the clinical and metabolic phenotype of women with polycystic ovary syndrome. Fertil Steril 103: 795-801, 2015.

AMATO MC, MAGISTRO A, GAMBINO G, VESCO R, GIORDANO C: Visceral adiposity index and DHEAS are useful markers of diabetes risk in women with polycystic ovary syndrome. Eur J Endocrinol 172: 79-88, 2015.

AZZIZ R, CARMINA E, DEWAILLY D, DIAMANTI-KANDARAKIS E, ESCOBAR-MORREALE HF, FUTTERWEIT W, JANSSEN OE, LEGRO RS, NORMAN RJ, TAYLOR AE, WITCHEL SF: The androgen excess and PCOS society criteria for polycystic ovary syndrome: the complete task force report. Fertil Steril 91: 456-488, 2009.

AZZIZ R, CHANG WY, STANCZYK FZ, WOODS K: Effect of bilateral oophorectomy on adrenocortical function in women with polycystic ovary syndrome. Fertil Steril 99: 599-609, 2013. 
CHEN J, SHEN S, TAN Y, XIA D, XIA Y, CAO Y, WANG W, WU X, WANG H, YI L, GAO Q, WANG Y: The correlation of aromatase activity and obesity in women with or without polycystic ovary syndrome. J Ovarian Res 8: 11-16, 2015.

CONWAY G, DEWAILlY D, DIAMANTI-KANDARAKIS E, ESCOBAR-MORREALE HF, FRANKS S, GAMBINERI A, KELESTIMUR F, MACUT D, MICIC D, PASQUALI R, PFEIFER M, PIGNATELLI D, PUGEAT M, YILDIZ BO; ESE PCOS SPECIAL INTEREST GROUP: The polycystic ovary syndrome: a position statement from the European Society of Endocrinology. Eur J Endocrinol 171: 1-29, 2014.

DAAN NM, JASPERS L, KOSTER MP, BROEKMANS FJ, DE RIJKE YB, FRANCO OH, LAVEN JS, KAVOUSI M, FAUSER BC: Androgen levels in women with various forms of ovarian dysfunction: associations with cardiometabolic features. Hum Reprod 30: 2376-2386, 2015.

DONG F, DENG D, CHEN H, CHENG W, LI Q, LUO R, DING S: Serum metabolomics study of polycystic ovary syndrome based on UPLC-QTOF-MS coupled with a pattern recognition approach. Anal Bioanal Chem 407: 4683-4695, 2015.

EBRAHIMI-MAMAGHANI M, SAGHAFI-ASL M, PIROUZPANAH S, ALIASGHARZADEH A, ALIASHRAFI S, REZAYI N, MEHRZAD-SADAGHIANI M: Association of insulin resistance with lipid profile, metabolic syndrome and hormonal aberrations in overweight or obese women with polycystic ovary syndrome. $J$ Health Popul Nutr 33: 157-167, 2015.

GEORGOPOULOS NA, PAPADAKIS E, ARMENI AK, KATSIKIS I, ROUPAS ND, PANIDIS D: Elevated serum androstenedione is associated with a more severe phenotype in women with polycystic ovary syndrome (PCOS). Hormones 13: 213-221, 2014.

GOODARZI MO, CARMINA E, R. AZZIZ R: DHEA, DHEAS and PCOS. J Steroid Biochem Mol Biol 145: 213-225, 2015.

GRIMMICHOVÁ T, VRBÍKOVÁ J, MATUCHA P, VONDRA K, VELDHUIS PP, JOHNSON ML: Fasting insulin pulsatile secretion in lean women with polycystic ovary syndrome. Physiol Res 57 (Suppl 1): S91-S98, 2008.

GUDUCU N, KUTAY SS, GORMUS U, KAVAK ZN, DUNDER I: High DHEAS/free testosterone ratio is related to better metabolic parameters in women with PCOS. Gynecol Endocrinol 31: 495-500, 2015.

KIM JJ, CHOI YM: Dyslipidemia in women with polycystic ovary syndrome. Obstet Gynecol Sci 56: 137-142, 2013.

KISCHE H, GROSS S, WALLASCHOFSKI H, VÖLZKE H, DÖRR M, NAUCK M, HARING R: Clinical correlates of sex hormones in women: The study of health in Pomerania. Metabolism 65: 1286-1296, 2016.

KUBOTA T: Update in polycystic ovary syndrome: new criteria of diagnosis and treatment in Japan. Reprod Med Biol 12: $71-77,2013$.

LAUGHLIN GA, GOODELL V, BARRET-CONNOR E: Extremes of endogenous testosterone are associated with increased risk of incident coronary events in older women. J Clin Endocrinol Metab 95: 740-747, 2010.

LAYEGH P, MOUSAVI Z, FARROKH TEHRANI D, PARIZADEH SM, KHAJEDALUEE M: Insulin resistance and endocrine-metabolic abnormalities in polycystic ovarian syndrome: Comparison between obese and non-obese PCOS patients. Int J Reprod Biomed 14: 263-270, 2016.

LEGRO RS, KUNSELMAN AR, DUNAIF A: Prevalence and predictors of dyslipidemia in women with polycystic ovary syndrome. Am J Med 111: 607-613, 2011.

LEGRO R: Obesity and PCOS: Implications for diagnosis and treatment. Semin Reprod Med 30: 496-506, 2012.

LERCHBAUM E, SCHWETZ V, RABE T, GIULIANI A, OBERMAYER-PIETSCH B: Hyperandrogenemia in Polycystic ovary syndrome: Exploration of the role of free testosterone and androstenedione in metabolic phenotype. PLoS One 9: e108263, 2014.

MARCH WA, MOOR WM, WILSON KJ, PHILLIPS DI, NORMAN RJ, DAVIES MJ. The prevalence of polycystic ovary syndrome in a community sample assessed under contrasting diagnostic criteria. Hum Reprod 25: 544-551, 2010.

MATHER KJ, KIM C, CHRISTOPHI CA, ARODA VR, KNOWLER WC, EDELSTEIN SE, FLOREZ JC, LABRIE F, KAHN SE, GOLDBERG RB, BARRETT-CONNOR E; DIABETES PREVENTION PROGRAM: Sex steroid hormones, sex hormone binding globulin, and diabetes incidence in the diabetes prevention program. $J$ Clin Endocrinol Metab 100: 3778-3786, 2015. 
MORAN C, ARRIAGA M, ARECHAVALETA-VELASCO F, MORAN S: Adrenal androgen excess and body mass index in polycystic ovary syndrome. J Clin Endocrinol Metab 100: 942-950, 2015.

NEHIR AYTAN A, BASTU E, DEMIRAL I, BULUT H, DOGAN M, BUYRU F: Relationship between hyperandrogenism, obesity, inflammation and polycystic ovary syndrome. Gynecol Endocrinol 32: 709-713, 2016.

PINOLA P, PILTONEN TT, PUURUNEN J, VANKY E, SUNDSTRÖM-POROMAA I, STENER-VICTORIN E, RUOKONEN A, PUUKKA K, TAPANAINEN JS, MORIN-PAPUNEN LC: Androgen profile through life in women with polycystic ovary syndrome: A Nordic Multicenter Collaboration Study. J Clin Endocrinol Metab 100: 3400-3407, 2015.

SCICCHITANO P, DENTAMARO I, CARBONARA R, BULZIS G, DACHILLE A, CAPUTO P, RICCARDI R, LOCOROTONDO M, MANDURINO C, MATTEO CICCONE M: Cardiovascular risk in women with PCOS. Int J Endocrinol Metab 10: 611-618, 2012.

SIRMANS SM, PATE KA: Epidemiology, diagnosis and management of polycystic ovary syndrome. Clin Epidemiol 6: $1-13,2014$.

SOISSON V, BRAILLY-TABARD S, HELMER C, ROUAUD O, ANCELIN ML, ZERHOUNI C, GUIOCHONMANTEL A, SCARABIN PY: A J-shaped association between plasma testosterone and risk of ischemic arterial event in elderly men: the French 3C cohort study. Maturitas 75: 282-288, 2013.

STENER-VICTORIN E, HOLM G, LABRIE F, NILSSON L, JANSON PO, OHLSSON C: Are there any sensitive and specific sex steroid markers for polycystic ovary syndrome? J Clin Endocrinol Metab 95: 810-819, 2010.

THE AMSTERDAM ESHRE/ASRM-SPONSORED 3RD PCOS CONSENSUS WORKSHOP GROUP: Consensus on women's health aspects of polycystic ovary syndrome (PCOS). Hum Reprod 27: 14-24, 2012.

THE JAPANESE SOCIETY OF OBSTETRICS AND GYNECOLOGY (JSOG): Constitute of reproductive endocrinology. Reports of a new diagnostic criteria of PCOS in Japan. Acta Obstet Gynaecol Jpn 59: 868-886, 2007.

THE ROTTERDAM ESHRE/ASRM-SPONSORED PCOS CONSENSUS WORKSHOP GROUP: Revised 2004 consensus on diagnostic criteria and long-term health risks related to polycystic ovary syndrome (PCOS). Hum Reprod 19: 41-47, 2004.

TORCHEN LC: Cardiometabolic risk in PCOS: More than a reproductive disorder. Curr Diab Rep 17: $137,2017$.

UNLU E, UNLU BS, YILDIZ Y, BEKER-ACAY M, KACAR E, TURAMANLAR O, TULMAC OB, SEVEN A, OZUGUZ U: Adrenal gland volume assessed by magnetic resonance imaging in women with polycystic ovary syndrome. Diag Interv Imag 97: 57-63, 2016.

YILDIZ BO, BOZDAG G, YAPICI Z, ESINLER I, YARALI H: Prevalence, phenotype and cardiometabolic risk of polycystic ovary syndrome under different diagnostic criteria. Hum Reprod 27: 3067-3073, 2012. 